silicon rods prepared by floating-zone melting in a vacuum or dry hydrogen.

The construction of nuclear fuel elements necessitates the welding of a variety of reactive metals and their alloys, such as zirconium, titanium, molybdenum and beryllium. Welding by electron bombardment heating ${ }^{2,3}$ has been found to give a better-quality weld for most metals than welding in an inert gas. P. Thomé (Centre D'Etudes Nucléaires de Saclay, France) described various electron guns in which the electron beam is focused to a target spot ranging in diameter from $0.01 \mathrm{~mm} .^{2}$ to $0.3 \mathrm{~mm}$. $^{2}$ for $20 \mathrm{~m}$.amp. and 1 amp. bombarding currents respectively at $20 \mathrm{kV}$. The beam of electrons passes through a hole in an earthed screen situated close to the components being welded, which are also at earth potential. This reduces the risk of glow discharge and arcing when fusion occurs. A further advantage is that there is no variation in the focus size with different shapes of pieces being welded. With the use of pulse heating, it is possible to apply a very high power to a small area and, if the speed of the work-piece is correctly related to the frequency of beam chopping, the weld consists of a series of overlapping spots. This permits a greater weld penetration and avoids the collapse of the sides of the weld due to metal flow which may happen in continuous welding, and also minimizes the area of recrystallization.

The general impression left by the conference was that in spite of some fundamental disadvantages, heating by electron bombardment will continue to play an important part in the science and technology of the more refractory metals and insulators, and will undoubtedly find other applications in high-temperature researches.

A. Calverley

${ }^{1}$ Davis, M., Calverley, A., and Lever, R. F., J. Appl. Phys., 27, 195 (1956).

${ }^{2}$ Stohr, J. A., le Vide, 13 (75), 163 (1958).

${ }^{3}$ Wyman, W. L., Welding J. Res. Supp. (Feb. 1958).

' Kelly, J. C., J. Sci. Instrum., 36, 89 (1959).

${ }^{5}$ Heywang, W., Z. Naturforsch., $11 a, 238$ (1956).

- Calverley, A., Davis, M., and Lever, R. F., J. Sci.Instrum., 34, 142 (1957).

'Allenden, D., J. Sci. Instrum., 36, 66 (1959).

8 Birbeck, F. E., and Calverley, A., J. Sci. Instrum. (in the press).

- Marshall, K. H. J. C., and Wickham, R., J. Sci. Instrum., 35, 121 (1958).

${ }^{10}$ Spacil, H. S., and Wulff, J., "Molybdenum", 13 (Amer. Soc. Metals, 1958).

${ }^{11}$ Mendelssohn, K., Canad. J. Phys., 34, 1319 (1956)

${ }^{12}$ Smith, H. R. "Vacuum Metallurgy", chap. 14 (Chapman and Hall, London, 1958).

${ }^{13}$ Calverley, A., and Rhys, D. W., Nature, 183, 599 (1959).

${ }^{14}$ Calverley, A., Proc. Phys. Soc., B, 70, 1040 (1957).

${ }^{15}$ Taylor, J. W., Metallurgia, Manc'ir., 50, 161 (1954).

${ }^{16}$ Heavens, O. S., J. Sci. Instrum., 36, 95 (1959).

\title{
TRANSISTOR CONVENTION AND EXHIBITION
}

\begin{abstract}
$\mathrm{A}^{\mathrm{N}}$ N International Convention on Transistors and Associated Semiconductor Devices was held at Earls Court, London, during May 21-27. The Convention was organized by the Electronics and Communications Section of the Institution of Electrical Engineers, which also promoted the International Transistor Exhibition held at Earls Court during the same period. The opening lectures were given by Prof. J. Bardeen, Dr. W. H. Brattain and Dr. W. B. Shockley. Some thirty further lectures were distributed among the eleven general and eleven specialist sessions that followed (mostly two at a time in parallel), brief summaries were presented of some eighty original papers, and some thirty-seven further original papers, of which either the full text or a brief digest had been previously circulated to those attending the Convention, were taken as read. The discussions at the sessions were rarely lively, perhaps because of the size and formality of the meetings and the acoustical defects of the halls (it was surprising not to find transistorized roving microphones provided to overcome this difficulty).

In his lecture Shockley considered the factors limiting the capabilities of the 'transistor-diode'-a silicon transistor operated without external base connexion up to voltages at which avalanche multiplication at the collector junction compensates for the initially low injection efficiency of the emitteras a fast switch for heavy currents. The tendency (due to the transverse ohmic voltage drop in the base region) for part of the emitter junction to draw to itself current from the rest could be overcome by switching on quickly enough after ensuring (by a suitable controlling wave-form) that the whole of the emitter is first just-sufficiently turned off. As an example an oscillogram was shown of a current
\end{abstract}

pulse rising to 50 amp. in 20 musec. across a load of about $0.5 \mathrm{ohm}$. In a separate paper Shockley showed that the minimization of the total capacitance of the emitter junction (also necessary in the device just considered) requires an optimum finite depletion layer thickness, on account of carrier storage in the layer.

Pritchard's lecture brought out the unsatisfactoriness of present commonly used equivalent circuits for transistors, particularly as regards the representation of the base resistance. Linvill generalized the Thevenin and Norton representations of a 2-terminal network to cover 2-ports, including the effects of internal 'contamination' generators representing, for example, noise or the dependence on temperature of d.c. parameters, and showed how the choice of an optimum source impedance could be solved generally for each of the last two situations. J. J. Sparkes showed that for some proportion of transistors the components $C_{b^{\prime} e}$ and $G_{b^{\prime} e}$ of the hybrid-pi equivalent circuit depart from their expected dependence on frequency, and suggested that the departures could be accounted for in terms of minority-carrier storage either in the emitter region or laterally in the base region. The effect could be correlated with anomalies in the switching characteristics.

The concept of charge-control (in contrast to current-control as generally considered typical of a transistor and voltage-control of a vacuum tube) was applied in detail by Beaufoy and by Kruithof to the quantitative description of the switching characteristics of transistors, and a generalized justification of the concept was presented in the lecture by Prof. Middlebrook.

Several papers set out to consider costs as related to some of the combinations of technologies now 
possible in the manufacture of transistors in large quantities. Although full agreement was lacking (not surprisingly, in view of the different markets and different degrees of reliability sought), a consensus of opinion seemed to favour one or other form of 'mesa' geometry, using diffusion alone or together with alloying, as the structure of the future. By the use of 'photo-lithographic' or other masking methods, large numbers of junctions could be completed simultaneously on each wafer of germanium or silicon, leaving a minimum of handling for the mounting and encapsulation of the individual transistors.

Atalla reported experiments on the electrical stability of silicon surfaces on which a thick (300 A. or more) oxide layer had been grown by exposure to water vapour or oxygen at about $1,000^{\circ} \mathrm{C}$. Such surfaces showed exceptional freedom from effects usually ascribed to changes in the density or nature of 'slow' electronic trapping states on the outer surface of the oxide; a reversible creep effect observed for a humid ambient in the leakage current of a $p-n$ junction having such a surface treatment was ascribed to the inversion layer induced in the underlying silicon on one side of the junction by the mobile ions electrolytically separated in the adsorbed water film as a result of the bias voltage applied across the junction. The oxide layer itself could withstand some $0.1 \mathrm{~V}$. per angstrom of thickness and should preferably be made thick enough to support the full breakdown voltage of the bulk $p-n$ junction.

The reliability of complete devices was considered explicitly in two papers, one of which showed that the activation energy for the ageing mechanism for a particular type of $p-n-p$ alloyed germanium unit was about $1 \cdot 2 \mathrm{eV}$., the end of useful life being brought about by the onset of rapid increase in leakage current and noise in a way characteristic of attack by water on the germanium surface.

A number of papers were devoted to the electrical parameters of semiconductor devices, and to their measurement. The frequency limitation (at about $1,000 \mathrm{Mc} / \mathrm{s}$.) set by the base width of transistors could be avoided by using single junction devices such as the variable-capacitance diode, and the diode proposed by Esaki in which the depletion layer is so thin as to allow tunnel effect to dominate the behaviour at low forward biases. It was necessary in high-frequency measurements to bring the point of measurement as close as possible to the envelope of the device. Measurements in the switching field were dominated by the charge-control approach, which uses rectangular wave-forms and $C R$ networks.

Sixty-four papers were devoted to applications; computers and switching applications were much the most prominent. Here the low power consumption and promised high reliability of transistors were seen to be of paramount importance, particularly as the earlier limitations on speed of operation were being largely overcome by developments in high-speed switching transistors. Chaplin illustrated the use of selected high-frequency alloy transistors (having a cut-off frequency of current gain of only about $10 \mathrm{Mc} . / \mathrm{s}$.) in the avalanche mode to generate pulses with times of rise of the millimicrosecond order.

Second in prominence to computers was the field of communications; here once again the transistor could reduce the size and power consumption of both mobile and fixed equipment and sometimes obviate the need for buildings. A paper by Grosser on space-division electronic telephone exchanges illustrated the important part that the pnpn structure can play as a substitute for a cross-point switch. Cave, Neu and Sim have used some specially made pointcontact germanium diodes as variable reactance modulators to control up to several hundred milliwatts of power at millimetre wave-lengths, with switching times as low as $3 \mathrm{~m} \mu \mathrm{sec}$.

Power diodes, silicon four-layer controlled rectifiers, and power transistors were all being applied to power supply and control. Besides emphasizing the small size and high efficiency of these devices, papers directed attention to the protection problems arising from the rapidity with which damage can occur.

In instrumentation, the diverse properties of semiconductors were being applied to a very wide range of applications. There were papers on the design of Hall effect multipliers, and also on single-crystal infra-red detectors using intermetallic compounds. Klein and Straub suggested that fast-neutron dosimetry could be carried out by electrically assessing the damage caused in germanium and silicon, and proposed an assembly of high-resistivity $p$-type germanium and lithium-6 for thermal-neutron dosimetry. McKenzie and Bromley dealt with the use of gold-germanium junctions as proportional particle spectrometers having a fast rise-time ( $<3$ musec.).

The exhibition was very well staged and paid due attention to research and up-to-date development as well as more conventional technology. The many pieces of equipment using transistors illustrated well their versatility and wide field of application. The exhibition showed also the extent to which industries ancillary to semiconductor technology, producing equipment such as furnaces, radio-frequency heaters, air-conditioning equipment, control instrumentation, etc., and supplies of high-purity material, are springing up. Among the newer semiconductor devices were mesa transistors from the United States and four-layer controlled rectifiers, and among the applications was a high-speed sampling oscilloscope exhibited by the Atomic Energy Research Establishment. The University of Birmingham showed experimental work on space-charge-limited currents in cadmium sulphide crystals. Associated Electrical Industries Research Laboratories showed some of the results of their investigation of etch-pits and other surface defects on germanium and silicon crystals, and of the diffusion of aluminium towards dislocations in silicon crystals.

This Convention was not intended as a continuation of the Reading-Amsterdam-Garmisch-Rochester series sponsored by the International Union of Puxe and Applied Physics, but rather as one to bring together the specialists from among the makers and users of transistors. It had something in common with the germanium and silicon materials and devices sessions of last year's Brussels conference. It is, however, to be hoped that the organizers of future conferences on transistors and related devices will take note of the very real risks of devaluation of the meetings caused both by overloading and by dilution of the programme with material that is either not pertinent enough or novel enough to those (generally already more or less expert in the subject-matter) most likely to benefit from the discussions.

F. F. Roberts

H. G. BASSETT 